\title{
Grade 3 Hepatotoxicity following Fulvestrant, Palbociclib, and Erdafitinib Therapy in a Patient with ER-Positive/PR-Negative/ HER2-Negative Metastatic Breast Cancer: A Case Report
}

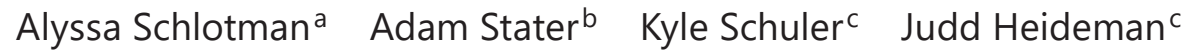 \\ Vandana Abramson ${ }^{a-c}$ \\ aVanderbilt University School of Medicine, Nashville, TN, USA; bVanderbilt-Ingram Cancer \\ Center, Nashville, TN, USA; 'Department of Medicine, Vanderbilt University Medical Center, \\ Nashville, TN, USA
}

\author{
Keywords \\ Hepatotoxicity · Breast cancer · Fulvestrant
}

\section{Abstract}

A 49-year-old woman with ER-positive/PR-negative/HER2-negative metastatic breast cancer experienced Grade 3 hepatotoxicity following initiation of a clinical trial of fulvestrant, palbociclib, and erdafitinib. Fulvestrant was determined to be the drug most likely responsible for this hepatotoxic effect. This case report details the timing and nature of this drug-induced liver injury, adding support to an area that has yet to be described adequately in the existing literature.

\section{Introduction}

A clinical trial is underway to study the safety and efficacy of fulvestrant, palbociclib, and erdafitinib in ER-positive, HER2-negative, FGFR-amplified metastatic breast cancer patients. Fulvestrant is a pure antiestrogen drug that is commonly used to treat postmenopausal women with hormone-receptor-positive metastatic breast cancer. Palbociclib is an inhibitor of cyclin-dependent kinases 4 and 6 that is used in combination with hormone therapy in ER+/HER2- postmenopausal metastatic breast cancer cases. Erdafitinib is an investigational pan-FGFR inhibitor. 
Schlotman et al.: Hepatotoxicity following Fulvestrant Therapy

Hepatotoxicity is a common side effect of cytotoxic chemotherapy, making the monitoring of liver function tests in patients on chemotherapy essential [1]. The drugs in this clinical trial have been associated with varying rates of hepatotoxicity. Fulvestrant has been associated with asymptomatic, transient, non-dose-limiting serum liver enzyme elevations in up to $15 \%$ of all patients. These elevations exceed 5 times the upper limit of normal in only $1-2 \%$ of all cases [2]. There is very little published work describing a detailed course and timing of these fulvestrant-induced liver injuries. One case report was found describing clinically apparent hepatotoxicity following fulvestrant therapy, which resolved within 2 weeks [3].

A review of the literature also reveals little support to clinically apparent liver injury attributable to palbociclib [4]. There has been one published report detailing two cases of pseudocirrhosis and liver-related death after 2-3 months of palbociclib therapy [5]. Another case report details elevated transaminases in a patient after three cycles of palbociclib [6]. Clinical trials of CDK4/6 inhibitors overall have shown rates of hepatotoxicity at less than $10 \%$, and the lowest rates have come with palbociclib specifically, with Grade 3/4 elevations in aspartate aminotransferase (AST) and alanine aminotransferase (ALT) reported in 3.3 and $2.3 \%$ of all patients, respectively [7].

There is little existing research on erdafitinib, but so far, the most common adverse events do not include hepatotoxicity [8]. One report details a case of dose-limiting hepatotoxicity occurring on cycle 1 day 15 (C1D15) of therapy. Another patient experienced Grade 3 elevations in AST and ALT on C1D14, which resolved after 8 days with dose interruption. There are largely no reports of Grade 4 hepatotoxicity with erdafitinib. Grade 3 elevations in ALT, AST, and alkaline phosphatase occurred in 3.7, 5.3, and $2.7 \%$ of all patients, respectively [9].

This report will detail a case of Grade 3 hepatotoxicity following initiation of this clinical trial of fulvestrant, palbociclib, and erdafitinib.

\section{Case Report}

A 49-year-old woman with ER+/PR-/HER2- breast cancer with metastasis to bone and liver began a clinical trial of palbociclib, fulvestrant, and erdafitinib on January 3, 2019 (C1D1). On this day, she received a 500-mg intramuscular injection of fulvestrant, one 125-mg tablet of palbociclib (to be taken once a day for 21 days then held for 7 days in a 28-day cycle), and two 4-mg tablets of erdafitinib (to be taken daily). Liver function tests were all within normal limits at this time. Two days later (C1D3), she became increasingly fatigued and developed nausea. The next day (C1D4), she began having watery pale diarrhea. On C1D5, she noted intense crampy abdominal pain in the right upper quadrant and mid-epigastric areas. She stopped the oral trial medications on C1D7. By this time, her symptoms had greatly improved, and she was left only with intermittent right upper quadrant pain. The next day (C1D8), she was found to have markedly elevated liver enzymes (Table 1), and she was admitted to Vanderbilt University Medical Center for further workup. She did not have altered mental status, jaundice, fever, or bleeding dysfunction.

Though medication side effect was suspected as the most likely etiology of the patient's acute liver injury, an extensive workup investigating other potential causes was performed. Hepatitis A panel, hepatitis B panel, hepatitis C IgG, and hepatitis D antibody were all negative. Acetaminophen and ethanol levels were within normal limits. Antinuclear antibody was negative. F-actin IgG was negative. CMV and EBV were negative. Abdominal ultrasound showed no evidence of cholecystolithiasis or biliary obstruction. 
Table 1. Serial liver function tests following chemotherapy initiation

\begin{tabular}{llccc}
\hline & $\begin{array}{l}\text { Total bilirubin, } \\
\text { mg/dL }\end{array}$ & $\begin{array}{l}\text { AST, } \\
\text { U/L }\end{array}$ & $\begin{array}{l}\text { ALT, } \\
\text { U/L }\end{array}$ & $\begin{array}{l}\text { Alkaline } \\
\text { phosphatase, U/L }\end{array}$ \\
\hline Normal range & $0.2-1.2$ & $5-40$ & $0-55$ & $40-150$ \\
December 18, 2018 & 0.4 & 23 & 21 & 79 \\
January 3, 2019 (Day 1) & 0.3 & 31 & 29 & 84 \\
January 10, 2019 (Day 8) 12:51 & 0.9 & 522 & 400 & 281 \\
January 10, 2019 (Day 8) 14:22 & 0.7 & 631 & 481 & 293 \\
January 11, 2019 (Day 9) & 1.0 & 698 & 848 & 312 \\
January 12, 2019 (Day 10) & 0.9 & 552 & 893 & 336 \\
January 13, 2019 (Day 11) & 0.7 & 247 & 636 & 313 \\
January 14, 2019 (Day 12) & 0.3 & 86 & 403 & 301 \\
January 15, 2019 (Day 13) & 0.4 & 50 & 305 & 274 \\
January 17, 2019 (Day 15) & 0.3 & 32 & 204 & 226 \\
\hline
\end{tabular}

Patient discontinued trial drugs on Day 7.

During the course of the patient's hospitalization, her liver enzymes continued to trend downward, and she remained asymptomatic. At its peak, AST reached a level of 698 (17.45 times the upper limit of normal, Grade 3 by NCI criteria) on C1D9, resolved to Grade 2 by C1D12, Grade 1 by C1D13, and returned to normal range by C1D15. ALT peaked at 893 (16.24 times the upper limit of normal, Grade 3 by NCI criteria) on C1D10 and resolved to Grade 2 by C1D15. Alkaline phosphatase peaked at $336 \mathrm{U} / \mathrm{L}$ (2.24 times the upper limit of normal, Grade 1 by NCI criteria) on C1D10 and remained Grade 1 throughout monitoring. Total bilirubin remained within normal limits throughout.

It was determined that the most likely culprit of this drug-induced liver injury (DILI) was fulvestrant. Of the three study drugs, it has the largest body of evidence supporting its history of hepatotoxic side effects. Furthermore, this patient reported symptom onset shortly after the fulvestrant injection, while her symptoms improved over the next week despite continuing to take erdafitinib and palbociclib. Furthermore, she had been treated previously with a 5 -month course of palbociclib with no adverse reaction or liver injury, thus making palbociclib unlikely to be the culprit. At 30 days' follow-up, the patient was without any new complaints or adverse events and had begun a new therapy.

\section{Discussion}

DILI is a common occurrence that can explain about $10 \%$ of all cases of acute hepatitis [10] and is one of the most common reasons for a drug to be withdrawn from the marketplace. Although the most commonly implicated drugs are acetaminophen and antibiotics, over 1,000 different medications have been shown to have the potential to cause DILI, including many chemotherapeutic drugs.

Various endocrine therapies for breast cancer have been shown to lead to liver injury, but the exact mechanisms for these processes are not well-understood. Fulvestrant is metabolized extensively by the liver via cytochrome P450 enzyme CYP3A4, but the exact mechanism of hepatocellular injury is uncertain. It has been suggested that toxic or immunogenic metabolic products of the drug may be responsible for hepatotoxicity [2]. Other similar hormonal treatments have also been implicated in hepatotoxicity. For example, the selective estrogen receptor modulator tamoxifen has a well-documented link to the development of 
Schlotman et al.: Hepatotoxicity following Fulvestrant Therapy

fatty liver and steatohepatitis with long-term therapy, attributed to its estrogenic effects on genetically predisposed livers [11]. Aromatase inhibitors that block estrogen synthesis have, like fulvestrant, been found to infrequently cause mild transient elevations in liver enzymes, but the exact mechanism of this injury has not been elucidated [12]. As the body of literature describing hepatotoxicity following these endocrine therapies grows, the description of the mechanisms behind these processes will be an important area of future research.

Although there is evidence supporting fulvestrant's potential for hepatotoxicity, there is very little existing data describing the timing and course of this liver injury in the current literature. With data available from this case report, this time course can be further elucidated. Symptoms of nausea and fatigue occurred 2 days after fulvestrant injection, and worsening right upper quadrant pain continued for the next 2 days before largely resolving by Day 7. AST elevation reached Grade 3 within 8 days of drug initiation, peaked on Day 9, and resolved to normal by Day 15. ALT elevation had reached Grade 3 by Day 8, peaked on Day 10 , and resolved to Grade 2 by Day 15. Alkaline phosphatase elevation reached Grade 1 by Day 8, peaked on Day 10, and remained Grade 1 by Day 15. Previous research had suggested that, although rare, fulvestrant could lead to severe elevations in liver enzymes. These data not only add support to that notion but provide evidence that this hepatotoxicity can be both severe and symptomatic. Our patient's course also suggests that this liver injury is reversible with discontinuation of the drug. This case enhances our understanding of an uncommon but serious complication of breast cancer treatment. As drug-induced hepatotoxicity gains more recognition in this field, we are better equipped to include it on differential diagnoses, understand what to expect as it progresses, and be proactive in treating it as we consider alternative treatment regimens for the underlying cancer.

\section{Statement of Ethics}

This report was conducted ethically with the consent of the patient.

\section{Disclosure Statement}

The authors have no conflicts of interest to declare.

\section{Author Contributions}

Alyssa Schlotman acquired and interpreted the data and drafted the work. Adam Stater, Kyle Schuler, and Judd Heideman acquired and interpreted the data and revised the work critically. Vandana Abramson conceived the original idea for the work, revised the work, and approved the final version to be published.

\section{References}

1 Grigorian A, O’Brien CB. Hepatotoxicity secondary to chemotherapy. J Clin Transl Hepatol. 2014;2(2):95-102.

2 National Library of Medicine. LiverTox: clinical and research information on drug-induced liver injury; Fulvestrant; 2019. Available from: http://livertox.nih.gov/Fulvestrant.htm.

3 Dziamski KM, Kalakonda A, Kohlitz P. A rare case of fulvestrant-associated jaundice and hepatotoxicity. Am J Ther. 2019;26(1):122-3.

4 National Library of Medicine. LiverTox: clinical and research information on drug-induced liver injury; Palbociclib; 2019. Available from: http://livertox.nih.gov/Palbociclib.htm. 
5 Vuppalanchi R, Saxena R, Storinolo AMV, Chalasani N. Pseudocirrhosis and liver failure in patients with metastatic breast cancer after treatment with palbociclib. Hepatology. 2017;65:1762-4.

6 Roberts BA, Ibrahim M, Stone E. Elevated liver function tests in a patient on palbociclib and fulvestrant. J Community Support Oncol. 2018;16(6):277-9.

7 Diéras V, Rugo HS, Schnell P, Gelmon K, Cristofanilli M, Loi S, et al. Long-term pooled safety analysis of palbociclib in combination with endocrine therapy for HR+/HER2- advanced breast cancer. J Natl Cancer Inst. 2018;111(4):419-30.

8 Nishina T, Takahashi S, Iwasawa R, Noguchi H, Aoki M, Doi T. Safety, pharmacokinetic, and pharmacodynamics of erdafitinib, a pan-fibroblast growth factor receptor (FGFR) tyrosine kinase inhibitor, in patients with advanced or refractory solid tumors. Invest New Drugs. 2018;36:424-34.

9 Erdafitinib Investigator's Brochure. Edition 7.29 August 2018.

10 Zimmerman HJ. Drug-induced liver disease. Clin Liver Dis. 2000;4(1):73-96.

11 National Library of Medicine. LiverTox: clinical and research information on drug-induced liver injury; Tamoxifen; 2019. Available from: http://livertox.nih.gov/Tamoxifen.htm.

12 National Library of Medicine. LiverTox: clinical and research information on drug-induced liver injury; Aromatase Inhibitors; 2019. Available from: http://livertox.nih.gov/AromataseInhibitors.htm. 